\title{
Cross-species genomics identifies DLG2 as a tumor suppressor in osteosarcoma
}

\author{
Yang W. Shao ${ }^{1} \cdot$ Geoffrey A. Wood $\mathbb{D}^{2} \cdot$ Jinchang Lu ${ }^{1,3} \cdot$ Qing-Lian Tang ${ }^{1,3} \cdot$ Jonathan Liu ${ }^{2} \cdot$ Sam Molyneux ${ }^{1}$. \\ Yan Chen ${ }^{1} \cdot$ Hui Fang ${ }^{1} \cdot$ Hibret Adissu ${ }^{2} \cdot$ Trevor McKee $^{1} \cdot$ Paul Waterhouse $^{1} \cdot$ Rama Khokha $^{1}$
}

Received: 19 March 2018 / Revised: 22 June 2018 / Accepted: 24 July 2018 / Published online: 9 August 2018

(c) The Author(s) 2018. This article is published with open access

\begin{abstract}
Leveraging the conserved cancer genomes across mammals has the potential to transform driver gene discovery in orphan cancers. Here, we combine cross-species genomics with validation across human-dog-mouse systems to uncover a new bone tumor suppressor gene. Comparative genomics of spontaneous human and dog osteosarcomas (OS) expose Disks Large Homolog 2 (DLG2) as a tumor suppressor candidate. DLG2 copy number loss occurs in $42 \%$ of human and $56 \%$ of canine OS. Functional validation through pertinent human and canine OS DLG2-deficient cell lines identifies a regulatory role of DLG2 in cell division, migration and tumorigenesis. Moreover, osteoblast-specific deletion of Dlg2 in a clinically relevant genetically engineered mouse model leads to acceleration of OS development, establishing DLG2 as a critical determinant of OS. This widely applicable cross-species approach serves as a platform to expedite the search of cancer drivers in rare human malignancies, offering new targets for cancer therapy.
\end{abstract}

\section{Introduction}

Extensive genomic alterations in orphan cancers make it difficult to identify driver mutations. Osteosarcoma (OS) presents a major challenge due to its chaotic genome and limited patient populations. Cytogenetic and genomic studies report a high degree of chromothripsis, chromosomal instability, copy number aberrations, and structural variations in OS [1-5]. Multiple patient cohorts have undergone aCGH amounting to 100 OS genomes, and whole-genome

These authors contributed equally: Yang W. Shao, Geoffrey A. Wood, Jinchang Lu.

Electronic supplementary material The online version of this article (https://doi.org/10.1038/s41388-018-0444-4) contains supplementary material, which is available to authorized users.

Rama Khokha

rama.khokha@utoronto.ca

1 Princess Margaret Cancer Centre, University of Toronto, Toronto, Ontario M5G 1L7, Canada

2 Department of Pathobiology, Ontario Veterinary College, University of Guelph, Guelph, Ontario N1G 2W1, Canada

3 Department of Orthopedic Oncology, the First Affiliated Hospital, Sun Yat-Sen Univeristy, 510080 Guangzhou, China sequencing was applied to 34 OS cases [6]. OS, the most common bone malignancy in adolescents, severely compromises the quality of life in children and adolescents. Further, despite improvements in orthopedic surgery and combination chemotherapy over the past 4 decades that have enhanced patient outcome, the 5-year survival rate of OS has plateaued at $\sim 65 \%$ [7]. New strategies are urgently required to uncover the molecular determinants of this cancer in order to design novel targeted therapies.

Naturally occurring canine cancers offer a cross-species approach to understanding the pathogenesis of human cancers due to the considerable homology between dog and human genomes. OS is one of the most frequent malignancies in dogs and shares remarkable clinical similarity with the human disease including radiological and histological features, and the pattern of metastatic spread. Compared to mice, dogs are relatively out-bred and share the same living environment as humans. Publication of highresolution dog reference genomes now permit a detailed genomic comparison between these species [8-11]. We created a novel comparative oncogenomics platform in this study, compiling high-resolution human and newly generated canine OS aCGH data, to identify known and novel drivers in bone cancer. Discs large homolog 2 (DLG2) was highly mutated in both human and dog, and was validated as a tumor suppressor through a workflow that spanned 
A

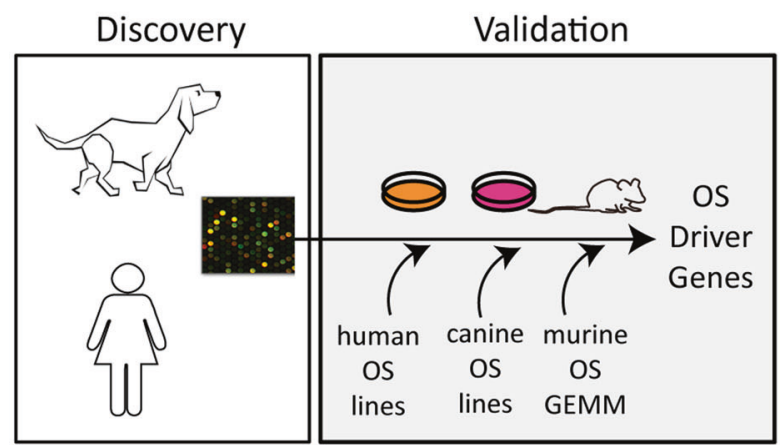

C
Human OS deletion landscape

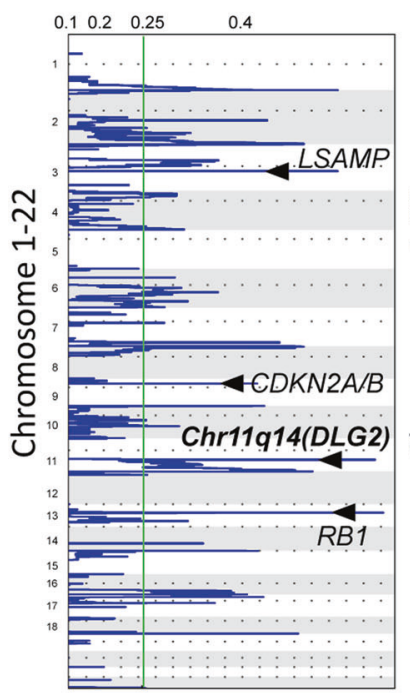

Canine OS deletion landscape

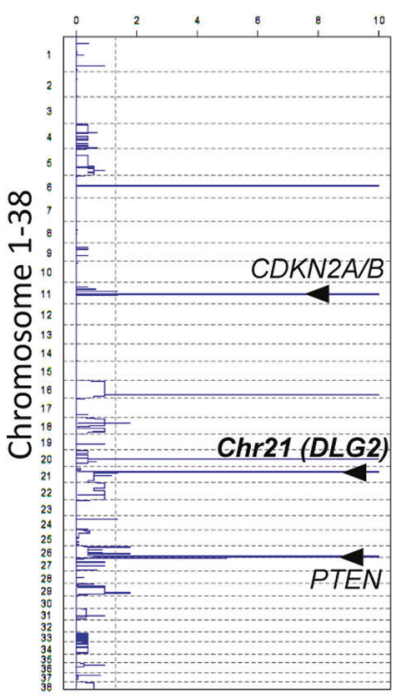

B

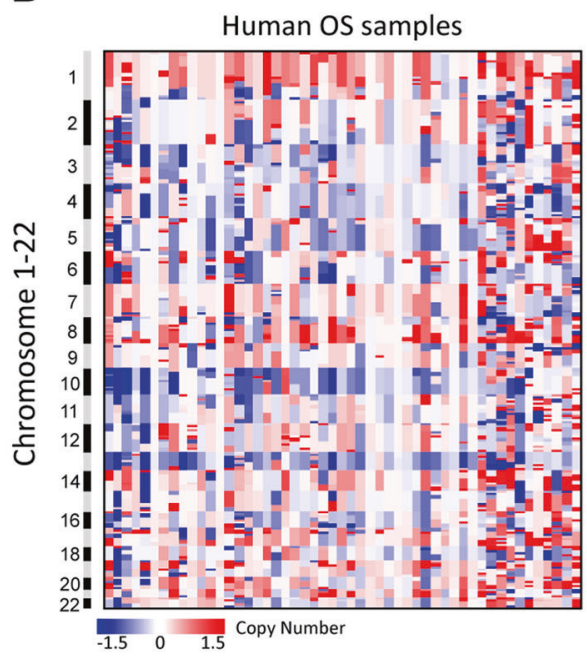

E
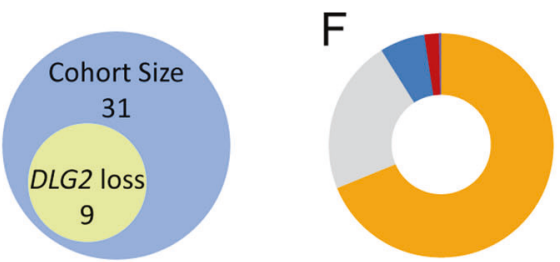

- Sub Missense

Sub Synonymous

- Sub Nonsense

- Indel Frameshift

- Indel In Frame

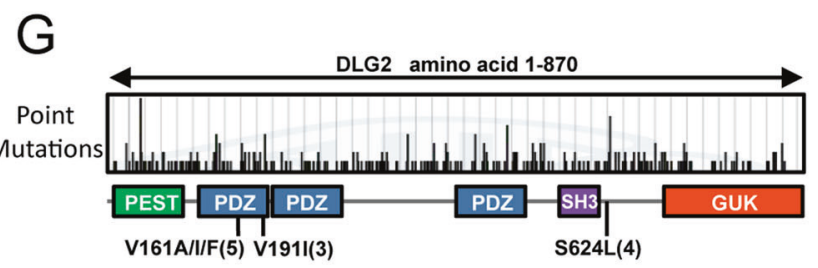

D
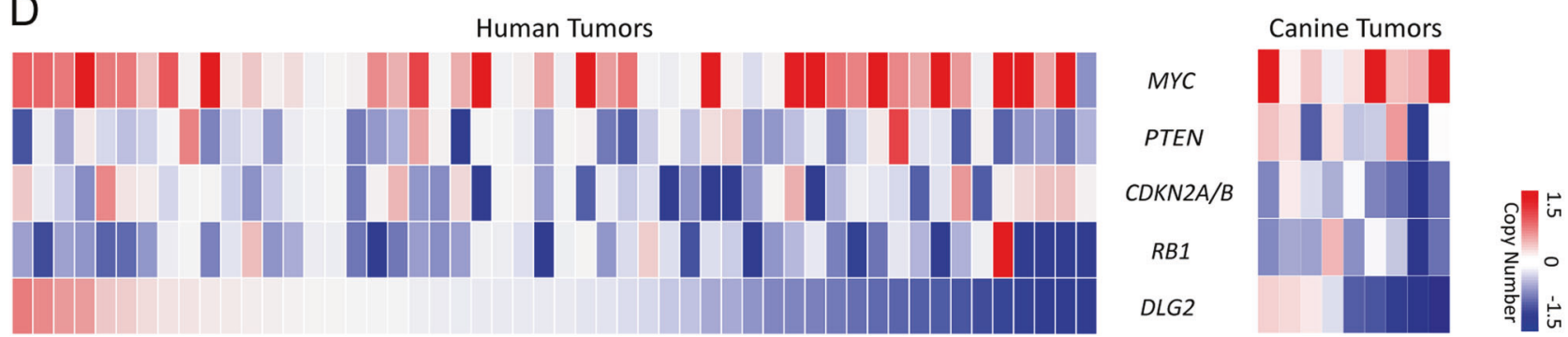

Fig. 1 Whole-genome landscape of CNAs in human and canine OS identifies DLG2 locus. a A schematic of cross-species oncogenomics strategy begins with comparison of curated human and new dog aCGH data on OS. Recurrent events serve as a filter to limit the number of candidate genes and selected new gene candidate undergoes biological validation through gain-of-function and loss-of-function studies designed to assess its impact on tumorigenic properties in vitro and oncogenic or tumor suppressor function in vivo. b Segmented CNA profiles of 52 human OS genome (left panel) and 9 canine OS genome (right panel) were mapped. $X$-axis presents individual samples. $Y$-axis

lists chromosomal locations from $\mathrm{Chr} 1$ to $\mathrm{Chr} 22$ for human, or $\mathrm{Chr} 1$ to Chr38 for dog. c Significant deletion regions calculated by GISTIC across the human and canine OS genome. Important genes in the peaks are labeled. $X$-axis: significant score, $Y$-axis: chromosomal locations. d GRID heat map showing copy number status of top mutated genes. Samples are ordered by DLG2 status. e Of 31 human OS samples, 9 exhibited DLG2 deletion in an independent cohort. $\mathbf{f}$ Pattern of point mutations in DLG2 genes based on data from COMSIC. Most mutations result in amino acid changes. g Mutation hot spots across DLG2 amino acid sequences 

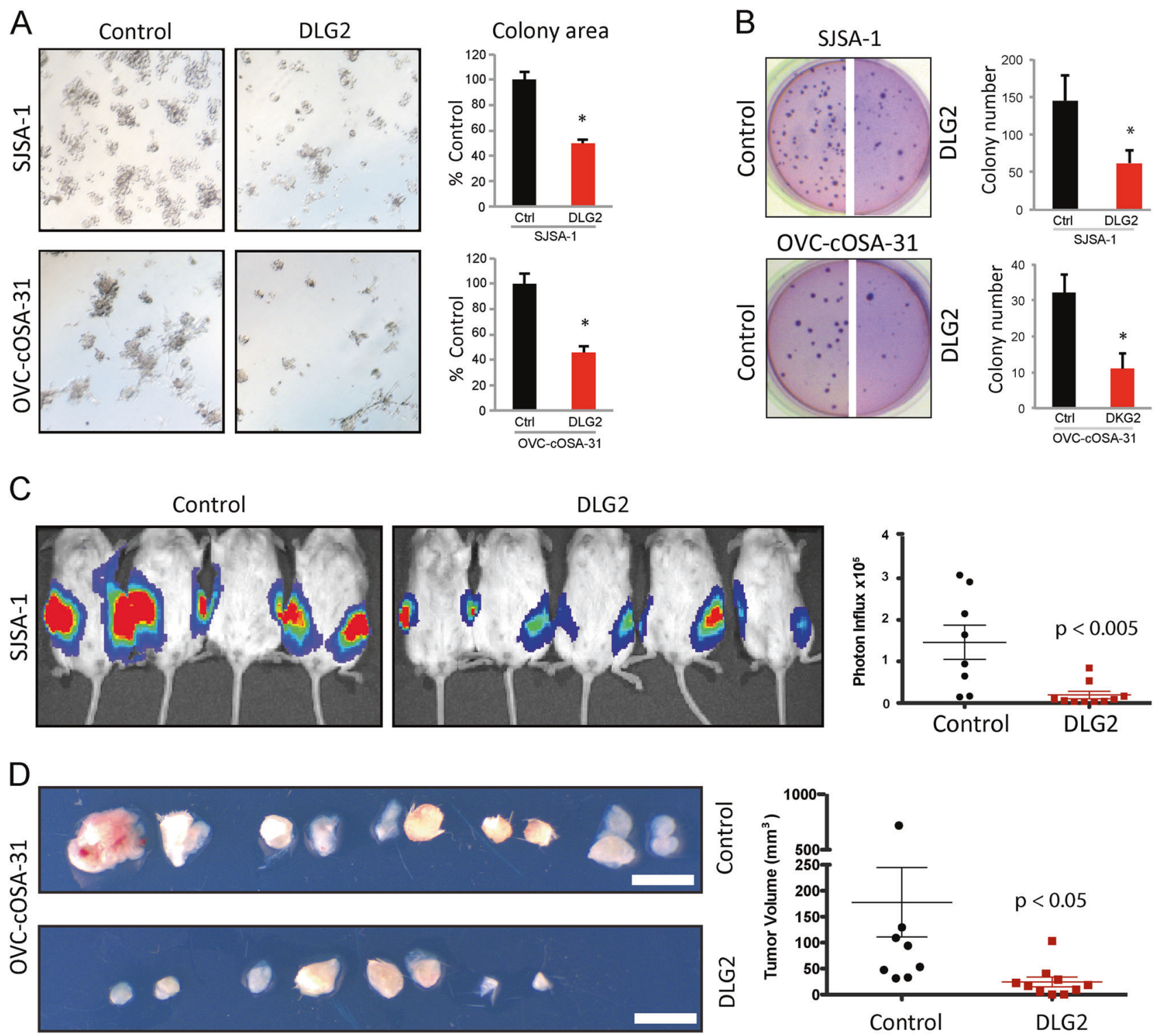

Fig. 2 DLG2 restoration hinders cell and tumor growth in in vitro 3D cultures and xenograft experiments. a Representative pictures of colonies in 3D-matrigel. DLG2 re-expression induced a significant reduction in both colony number and size. Quantification of colony area is shown on the right. b Representative soft agar images and their respective quantifications. DLG2 restoration reduces colony forming capacities. c NOD-SCID mice injected with luciferase- expressing SJSA-1 parental (left) and DLG2 (right) cells imaged at 3 weeks post

human and canine cell lines, and a clinically relevant murine model of OS.

\section{Results and discussion}

To create a comparative oncogenomics platform (Fig. 1a), we selected a published dataset of 52 human OS samples which contain 40 tumor specimens and 12 OS cell lines (GSE12789). In parallel, 9 canine OS specimens injection ( $n=10$ per group). Photon influx value was used as a surrogate marker for tumor size. Photon influx of each tumor was quantified and average values are shown on the right. $\mathbf{d}$ Tumors taken from NOD-SCID mice 4 weeks after innoculation with OVC-COSA31 parental (left) and DLG2 (right) canine OS cells ( $n=10$ per group). Tumor size was calculated and is shown on the right. White bar represents $1 \mathrm{~cm}$. Values shown are the mean $\pm S D$ of three separate determinations. ${ }^{*} P<0.05$ by two-tailed Student's $t$ test

representing a number of dog breeds were subjected to aCGH (Supplemental Table S1). Among the multiple tools used to segment the human and canine data, we found the greatest concordance between Circular Binary Segmentation (CBS) and Gain and Loss Analysis of DNA (GLAD); these were used to define the copy number profile $[12,13]$. Segmented data were next analyzed by GISTIC (FDR $Q$ value $<0.1)$ to shortlist recurrent genomic regions for each species [14]. While candidate genes in human were directly produced by GISTIC, those in dog were pinpointed 

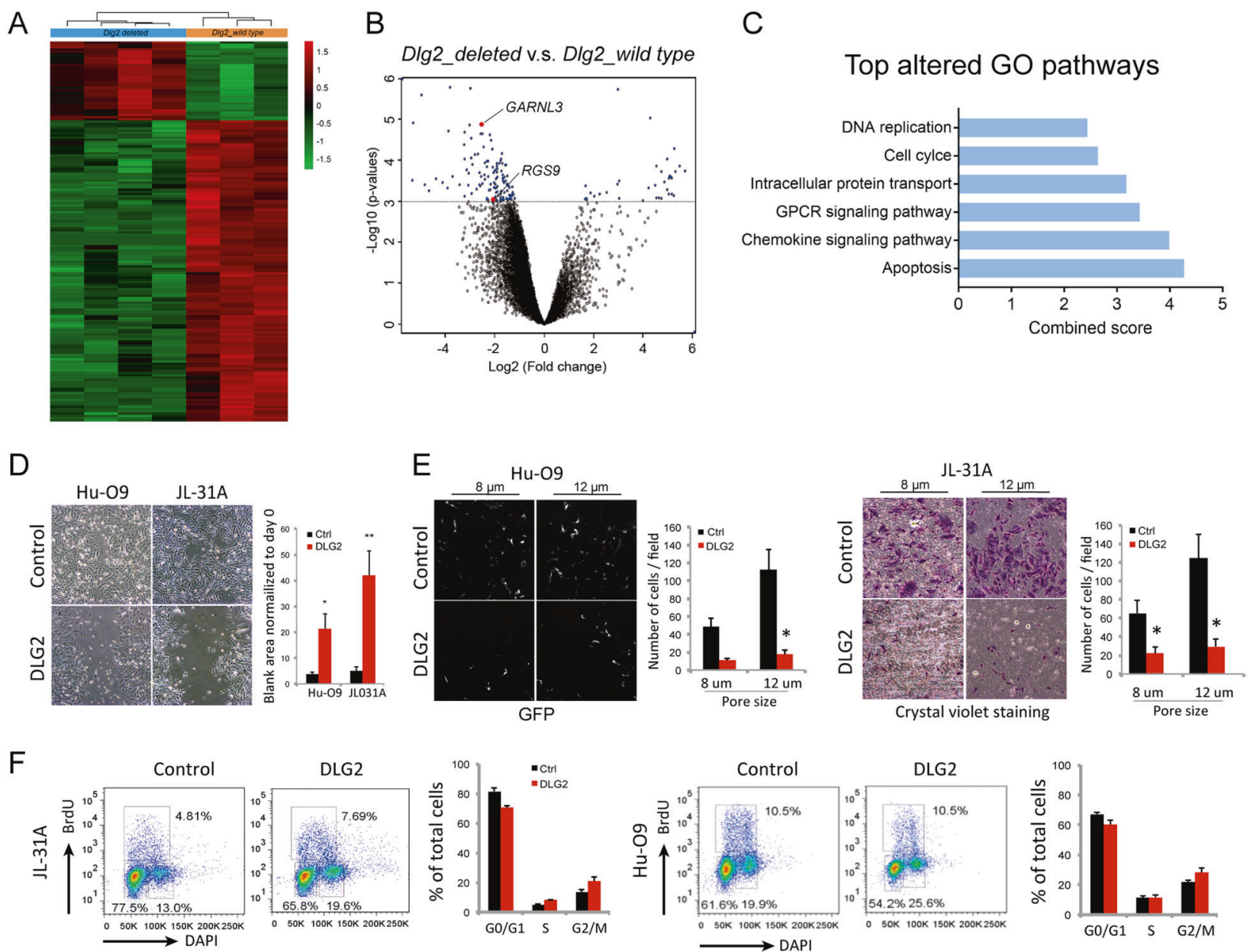

Fig. 3 DLG2 is involved in small GTPase activity and its restoration reduces migratory capacities. a Differentially expressed probes/genes in Dlg2-deleted ( $n=4$, blue) vs. Dlg2-wild-type $(n=3$, orange) groups. b Volcano plots show differential probes/genes identified using $T$-test with FDR correction. c Top altered pathways were analyzed using Enrichr online tool. G- protein-coupled receptor protein signaling pathway was among the top significant pathways. d Representative images of HuO9 and OVC-COSA-31 control and DLG2-reexpressing cells $24 \mathrm{~h}$ after a scratch-wound. Quantification of blank areas is shown on the right. e Representative images of $\mathrm{Hu}-\mathrm{O} 9$ and OVC-COSA-31 cells $24 \mathrm{~h}$ after seeding in transwells. Transwell insert

pore size of 8 and $12 \mu \mathrm{m}$ were used. Quantification of cells that migrated through the transwell is shown on the right. $\mathbf{f}$ Cell cycle analysis showing DLG2 induced cell cycle changes in OVC-COSA-31 and $\mathrm{Hu}-\mathrm{O} 9$ cells cultured in ultra-low attachment plate. DAPI ( $x$-axis) was used to determine DNA content and $\operatorname{BrdU}$ ( $Y$-axis) was used to determine nucleotide uptake. DLG2 restoration resulted in significant accumulation of cells in G2/M and S phases. Quantification of cells at specific stages of the cell cycle is shown on the right. Values shown are the mean \pm SD of three separate determinations. $* P<0.05$ by twotailed Student's $t$ test

through cross species synteny matching of 38 canine chromosomes to the human reference genome, hg19. Human and canine OS genomes contained high levels of chromosomal instability marked by frequent whole chromosomal arm gains and losses (Fig. 1b). Mutational frequency of recurrent CNAs across samples showed that among the top eight highly significant genes, six were common between human and canine OS (Fig. 1c and Supplemental Fig. 1A), providing proof-of-principle for our approach. These included known oncogenes $(M Y C)$ and tumor suppressors $(C D K N 2 A / B, R B 1$, and PTEN) indicating the high level of disease similarity and the overlapping mutational landscape across these species [15-
18]. We recorded a novel focal deletion in Chr11q14 across multiple cases, harboring potential tumor suppressor gene (s) (Fig. 1c). Specifically, 42\% (22/52; Chr11q14) of human OS and 55.6\% (5/9; Chr21) of canine OS exhibited this focal loss (Supplemental Fig. 1B). This frequency is consistent with that reported for next-generation sequencing of 34 human OS cases $(52.9 \%, 18 / 34)$ [6], although its biological significance was not pursued. We noted that $D L G 2$ is the only gene residing in the focal deletion in human (chr11: 83835663-84011662: $180 \mathrm{~kb}$ ) and in dog (chr21:17457665-17858164: $400 \mathrm{~kb}$ ). The relative distribution of $D L G 2$ deletion and other common alterations across human and dog OS is shown in Fig. 1d. 
A

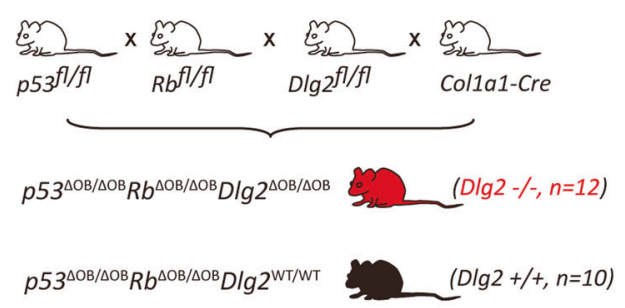

B

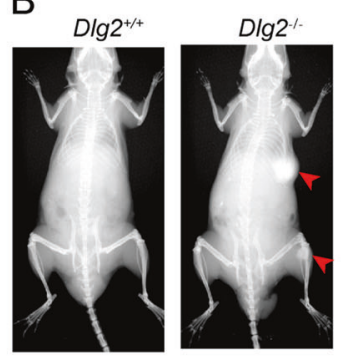

C

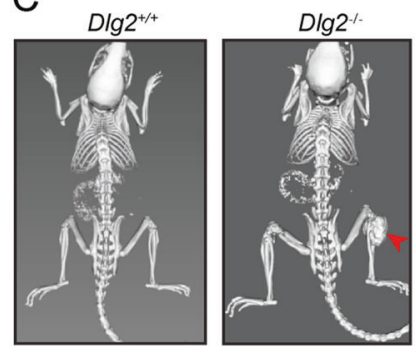

D

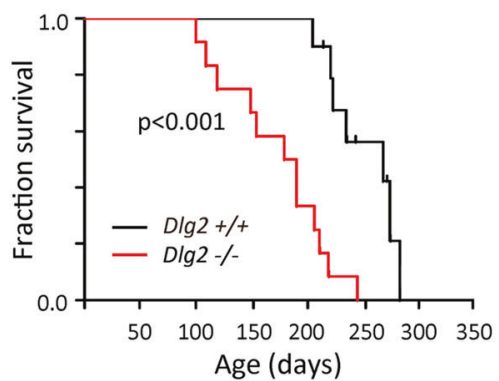

$\mathrm{E}$

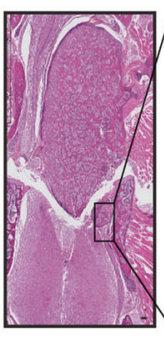

$\mathrm{F}$

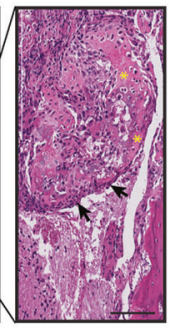

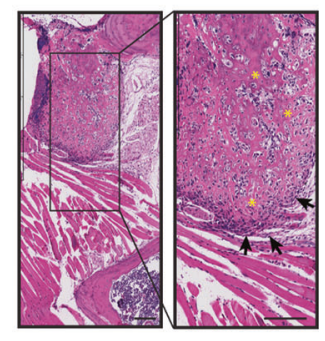

G

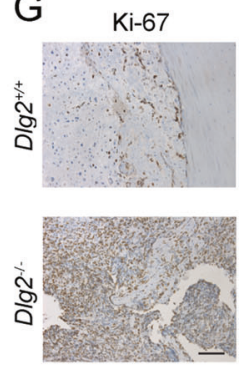

Fig. 4 Osteoblast-specific Dlg2 knockout reduces overall survival and accelerates tumor onset. a Breeding strategy for generating osteoblastspecific compound deletion of Dlg2, p53, and Rb1. b Representative X-ray and c MicroCT images. At 22 weeks of age, Dlg2 wild type mice show no visible signs of tumor while Dlg2 conditional knockout mice developed tumors on tibia and rib. d Kaplan-Meier curve of mice with different Dlg2 status. Dlg2 homozygous conditional knockout shortens overall survival compared to Dlg2 wild type. e H\&E images of a thoracic vertebra osteosarcoma. At higher magnification, osteosarcoma cells (arrows) and osteoid deposition (asterisk) were seen invasive fronts. f $\mathrm{H} \& \mathrm{E}$ images of a femoral osteosarcoma located on the Ilium. Note the plump tumorous osteoblasts (arrows) and massive osteoid deposition (asterisk). g Ki-67 staining of indicated tumor tissues. Scale bar: $100 \mu \mathrm{m}$
We then acquired an independent cohort of 31 human OS samples to examine the $D L G 2$ status using genomic qPCR (courtesy of Dr. David Malkin). Of 31 samples, 9 displayed $D L G 2$ deletion (29\%; Fig. 1e), providing a validation in this independent patient cohort. Next, interrogation of the Catalogue of Somatic Mutations in Cancer (COSMIC) database exposed $D L G 2$ to be highly mutated in a variety of human cancers where most of the mutations resulted in drastic amino acid changes (Fig. If and Supplemental Table 2) [19]. DLG2 is a member of the Membrane-Associated Guanylate Kinase (MAGUK) protein family that is characterized by concatenations of PDZ, SH3 and GUK domains. These domains enable MAGUKs to function as scaffold proteins that orchestrate the assembly of multiple signal transduction networks [20]. The mammalian DLG family of proteins are primarily known for their role in epithelial polarity and polarity during cell division [21]. Several mutational hotspots were seen: V161 and V191 within the first PDZ domain; and S624 in the connector sequence adjacent to the $\mathrm{SH} 3$ domain (Fig. $1 \mathrm{~g}$ ).

To examine the impact of restoring DLG2 expression on tumorigenic properties, we sought relevant human and canine cell lines that naturally harbored DLG2 deletions. Data from GSE12789 and GSE36003 revealed that DLG2 was deleted in SAOS-2, SJSA-1 and 2 primary OS cell lines
(HuO-3N1 \& HuO9), while DLG2 loss was also detected by aCGH analysis in a canine OS cell line that we generated in house (OVC-cOSA-31); Supplemental Fig. 1C). Consistently, endogenous DLG2 protein expression was very low in these cells (Supplemental Fig. 1D). A human DLG2 expression vector was then constructed for the dominant, longest isoform1 (Supplemental Fig. 1D). DLG2 expression did not affect proliferation in normal or low-serum conditions (Supplemental Fig. 2A). However, when tested in anchorage-independent soft agar and 3D-Matrigel assays, DLG2 overexpression in SJSA-1 or OVC-COSA-31 cells significantly reduced their colony formation capacity (Fig. 2a, b). HuO9 and SAOS-2 did not grow in anchorageindependent conditions. We then tested the effect of DLG2 restoration in vivo. Luciferase-tagged [22], parental SJSA-1 and OVC-COSA-31 cell lines formed xenografts when injected subcutaneously into NOD-SCID mice, whereas DLG2 restored cell lines showed slower xenograft growth, resulting in smaller tumors (Fig. 2c, d).

We next investigated the gene expression profiles associated with DLG2 deletion in canine OS. Microarrays generated from 7 aCGH-matched canine primary tumors (4 $D L G 2$ deleted, 3 DLG2 wild type) showed 167 differentially expressed probes/genes (DEGs) in the two groups (Fig. 3a, b, Supplemental Table 3). Interestingly, G protein- 
coupled receptor (GPCR) signaling was one of the top altered pathways (Fig. 3c). The functional interactions between PDZ domain-containing proteins such as DLG1 and DLG4, and GPCRs have been implicated in many diseases including cancer [23]. Indeed, biological process prediction of DLG2 using Integrative Multi-species Prediction (IMP) database (http://imp.princeton.edu) showed that DLG2 is also involved in GPCR signaling pathway (GO term ID: GO:0086103, GO:0007186, GO:0008277). Importantly, GTPase-activating proteins RGS9 (Regulator of G-protein signaling 9) [24] and GARNL3 (GTPase activating Rap/RanGAP domain-like 3) [25] called as GAPs were among the most downregulated genes between $D L G 2$ deleted and $D L G 2$-wild-type groups (Fig. 3b). GAPs are a family of regulatory proteins that bind to activated $G$ proteins and result in "switching off" of GTPase signaling [26]. Therefore, RGS9 and GARNL3 downregulation in DLG2deleted tumor may lead to increased GTPase signaling activity.

Small GTPase signaling pathway, such as Rho and Ran, has been previously implicated in cytoskeletal reorganization and migration [27]. We therefore performed scratch-wound and transwell migration assays. DLG2expressing cell lines showed significantly decreased motility in both assays compared to their respective parental control cells (Fig. 3d, e). This phenotype is in agreement with our gene expression data indicating a negative regulatory role for DLG2 in the GTPase signaling pathway, which in turn influences human and canine OS cell migration. Since GTPase signaling also orchestrates multiple stages of the cell cycle, we asked whether DLG2 status affects the cell cycle using flow cytometry [28]. We observed more cells in either $\mathrm{G} 2 / \mathrm{M}$ or $\mathrm{S}$ phases when DLG2-re-expressing cells were grown under lowattachment conditions (Fig. 3f) but not when grown as a monolayer (data not shown). This observation together with slower proliferation under 3D, but not 2D conditions (Fig. 2a, b, and Supplemental Fig. 2A) suggests that DLG2 participates in regulating cell cycle progression. It is likely that the tumor suppressor function of DLG2 is mediated in part through modulating the small GTPase signaling pathway.

The single Drosophila DLG homolog is a tumor suppressor [29]. To examine whether mammalian DLG2 functions as a tumor suppressor, we utilized a genetically engineered mouse model (GEMM) of OS. $R B$ and TP53 mutations are the most common events in OS and germline mutations also predispose humans to this cancer. It has been shown that combined osteoblast-specific deletion of $p 53$ and $R b$, achieved through the Collagen Type 1 Alpha-1 (Collal) promoter-driven Cre recombination in mice leads to spontaneous OS development [30, 31]. We bred Dlg2-floxed mice into the above clinically relevant model to generate triple osteoblast-specific deletions
( $p 53^{\Delta \mathrm{OB} / \Delta \mathrm{OB}} R b^{\Delta \mathrm{OB} / \Delta \mathrm{OB}} D \lg 2^{\Delta \mathrm{OB} / \Delta \mathrm{OB}}: D \lg 2^{-/-}$, Fig. 4 a). Dlg 2 homozygous deletion significantly accelerated tumor development and shortened survival in mice (Fig. 4b-d). Specifically, the median lifespan for the $D \lg 2^{-/-}$cohort was 184 days vs. 239 days in the control group $\left(p 53^{\Delta \mathrm{OB} / \Delta \mathrm{OB}} R b^{\Delta \mathrm{OB} /}\right.$ $\left.{ }^{\mathrm{OB}} D \lg 2^{\mathrm{WT} / \mathrm{WT}}: D \lg 2^{+/+}\right)$. The anatomical distribution and histological features of OS tumors developed in $\mathrm{Dlg} 2^{-/-}$mice remained comparable to littermate controls. The abundant osteoid deposition at the invasive the front of thoracic vertebra osteosarcoma (Fig. 4e) illustrates the aggressive nature, osteoblastic nature of tumors developed in these mice. Histological features of osteosarcoma, such as plump tumorous osteoblasts and copious amount of osteoid were also seen in tumors that developed at different sites in the Dlg2-null mice (Fig. 4f). Moreover, tumor cells derived from Dlg2-deficient mice were more proliferative than those of wild-type mice, as indicated by Ki-67 staining (Fig. 4g).

Altogether, through cross-species oncogenomics we have uncovered $D L G 2$ deletion to be a highly frequent event in human $(42 \%)$ and dog (56\%) OS. We extended our crossspecies approach to molecular and functional analyses of DLG2 by utilizing pertinent human and dog OS cell lines as well as p53/Rb-driven OS GEMM. These series of studies establish DLG2 as a bone tumor suppressor and provide insight into its involvement in GTPase signaling and regulation of cell cycle and proliferation in a $3 \mathrm{D}$ tissue culture model. This work presents a blue print for cross-species comparative genomics platform as a useful tool for driver gene discovery in rare human cancers.

\section{Methods}

\section{aCGH analysis}

Published human OS genomic datasets on Gene Expression Omnibus (GEO) were selected (GSE12789). Canine OS specimens were obtained from University of Guelph veterinarian clinics. Genomic DNA was extracted using phenol chloroform and treated with RNase A (Invitrogen) for $1 \mathrm{~h}$ at $37^{\circ} \mathrm{C}$. Canine aCGH assay was performed by SurePrint G3 Canine CGH Microarray $4 \times 180 \mathrm{~K}$ (Agilent) according to manufacturers' specifications at the University Health Network Microarray Centre. CBS and GLAD were used for segmentation of the genome and GISTIC was used to determine the common altered region and the significance of genetic events. Canine aCGH data were deposited in the GEO database (GSE111637).

\section{Gene expression microarray analysis}

Total RNA from canine OS tumors was prepared using the RNeasy mini-kit (QIAGEN), RNA concentration and 
integrity were assessed using Nanodrop 2000 and Bioanalyzer (Thermo Scientific and Agilent). Gene expression microarray on Canine (V2) Gene Expression Microarray, $4 \times 44 \mathrm{k}$ (Agilent) were performed at the University Health Network Microarray Centre. Multi-array average (RMA) normalization was used across the sample sets and batch correction was performed. Differentially expressed genes were identified using $T$-test with FDR correction $(P<0.05$ and FDR $<0.1)$ or Samtools. Analyses were performed using R (version 2.82), Agilent Genespring GX (version 11) or BRBArrayTools. Gene ontology and pathway analysis were performed using Enrichr. Total Ensemble genes were used as background. Microarray data were deposited in the GEO database (GSE111638).

\section{Mice}

Osteoblast-specific Dlg2 knockout OS mouse model $\left(p 53^{\triangle O B / \triangle O B} R b^{\triangle O B / \triangle O B} D \lg 2^{\triangle O B / \triangle O B}\right.$ ) was generated by breeding Dlg $g^{\text {flox/+ }} \quad\left(D \lg 2^{\text {tmla(EUCOMM }) W t s i}\right.$, obtained from Wellcome Trust Sanger Institute) with Collal-Cre$p 53^{\text {flox/flox }} R b^{\text {flox/flox }}(n=12) . p 53^{\triangle O B / \triangle O B} R b^{\Delta O B / \triangle O B} D \lg 2^{\text {wt } / w t}$ littermates $(n=10)$ were used as the control. X-ray and MicroCT scan were carried out as previously reported [28]. For xenograft experiments, 8-week-old female recipient non-obese diabetic/severe combined immunodeficiency (NOD-SCID) mice were obtained from Charles River Laboratories. Mice were assigned to experimental groups by stratified randomization according to body weight. OVCCOSA-31 and luciferase-expressing SJSA-1 cells were injected subcutaneously on both flanks ( $n=10$ per group) and monitored weekly for 4-8 weeks by electronic caliper or bioluminescence imaging using Xenogen IVIS Imaging System 100 (STTARR facility, UHN). Investigators who measured and analyzed the samples were blind to the group information.

\section{Statistics}

Two-tailed Student's $t$-test was used to determine if two sets of normally distributed data are significantly different from each other. Biological replicates were performed independently at least three times. Log-rank test was performed for survival curves. For all studies, $P<0.05$ was considered significant. Specialized analyses for bioinformatic data, including aCGH analysis and gene expression microarray analysis, were performed using dedicated tools mentioned above.

\section{Study approval}

All animal protocols were approved by the University Health Network Animal Care Committee and performed in accordance with the standards of the Canadian Council on Animal Care. Mice were housed in the Princess Margaret Hospital Animal Care Facility. User protocol AUP 849.1 was strictly followed. Written informed consent was obtained from the patients or their guardians before sample collection.

Acknowledgements We thank all members of our laboratory for helpful discussions.

Funding This study is supported by funding from the Canadian Institutes of Health Research (CIHR), Ontario Institute for Cancer Research (OICR), and Global Leadership Round in Genomics and Life Sciences (GL2) to RK and the OVC Pet Trust to GW. J.Lu is also supported by National Natural Science Foundation of China (81702665), National Postdoctoral Program for Innovative Talents (BX201600196) and China Postdoctoral Science Foundation (2017M610571).

\section{Compliance with ethical standards}

Conflict of interest The authors declare that they have no conflict of interest.

Open Access This article is licensed under a Creative Commons Attribution 4.0 International License, which permits use, sharing, adaptation, distribution and reproduction in any medium or format, as long as you give appropriate credit to the original author(s) and the source, provide a link to the Creative Commons license, and indicate if changes were made. The images or other third party material in this article are included in the article's Creative Commons license, unless indicated otherwise in a credit line to the material. If material is not included in the article's Creative Commons license and your intended use is not permitted by statutory regulation or exceeds the permitted use, you will need to obtain permission directly from the copyright holder. To view a copy of this license, visit http://creativecommons. org/licenses/by/4.0/.

\section{References}

1. dos Santos Aguiar S, de Jesus Girotto Zambaldi L, dos Santos AM, Pinto W Jr, Brandalise SR. Comparative genomic hybridization analysis of abnormalities in chromosome 21 in childhood osteosarcoma. Cancer Genet Cytogenet. 2007;175:35-40.

2. Nathrath MH, Kuosaite V, Rosemann M, Kremer M, Poremba C, Wakana S, et al. Two novel tumor suppressor gene loci on chromosome $6 \mathrm{q}$ and $15 \mathrm{q}$ in human osteosarcoma identified through comparative study of allelic imbalances in mouse and man. Oncogene. 2002;21:5975-80.

3. Kuijjer ML, Rydbeck H, Kresse SH, Buddingh EP, Lid AB, Roelofs $\mathrm{H}$, et al. Identification of osteosarcoma driver genes by integrative analysis of copy number and gene expression data. Genes Chromosomes Cancer. 2012;51:696-706.

4. Chen X, Bracht JR, Goldman AD, Dolzhenko E, Clay DM, Swart EC, et al. The architecture of a scrambled genome reveals massive levels of genomic rearrangement during development. Cell. 2014;158:1187-98.

5. Stephens PJ, Greenman CD, Fu B, Yang F, Bignell GR, Mudie LJ, et al. Massive genomic rearrangement acquired in a single catastrophic event during cancer development. Cell. 2011;144:27-40.

6. Chen X, Bahrami A, Pappo A, Easton J, Dalton J, Hedlund E, et al. Recurrent somatic structural variations contribute to tumorigenesis in pediatric osteosarcoma. Cell Rep. 2014;7:104-12. 
7. Aljubran AH, Griffin A, Pintilie M, Blackstein M. Osteosarcoma in adolescents and adults: survival analysis with and without lung metastases. Ann Oncol. 2009;20:1136-41.

8. Tsai PC, Breen M. Array-based comparative genomic hybridization-guided identification of reference genes for normalization of real-time quantitative polymerase chain reaction assay data for lymphomas, histiocytic sarcomas, and osteosarcomas of dogs. Am J Vet Res. 2012;73:1335-43.

9. Angstadt AY, Thayanithy V, Subramanian S, Modiano JF, Breen M. A genome-wide approach to comparative oncology: highresolution oligonucleotide aCGH of canine and human osteosarcoma pinpoints shared microaberrations. Cancer Genet. 2012;205:572-87.

10. Angstadt AY, Motsinger-Reif A, Thomas R, Kisseberth WC, Guillermo Couto C, Duval DL, et al. Characterization of canine osteosarcoma by array comparative genomic hybridization and RT-qPCR: signatures of genomic imbalance in canine osteosarcoma parallel the human counterpart. Genes Chromosomes Cancer. 2011;50:859-74.

11. Scott MC, Sarver AL, Gavin KJ, Thayanithy V, Getzy DM, Newman RA, et al. Molecular subtypes of osteosarcoma identified by reducing tumor heterogeneity through an interspecies comparative approach. Bone. 2011;49:356-67.

12. Venkatraman ES, Olshen AB. A faster circular binary segmentation algorithm for the analysis of array CGH data. Bioinformatics. 2007;23:657-63.

13. Hupe P, Stransky N, Thiery JP, Radvanyi F, Barillot E. Analysis of array CGH data: from signal ratio to gain and loss of DNA regions. Bioinformatics. 2004;20:3413-22.

14. Beroukhim R, Getz G, Nghiemphu L, Barretina J, Hsueh T, Linhart D, et al. Assessing the significance of chromosomal aberrations in cancer: methodology and application to glioma. Proc Natl Acad Sci USA. 2007;104:20007-12.

15. Shimizu T, Ishikawa T, Sugihara E, Kuninaka S, Miyamoto T, Mabuchi Y, et al. c-MYC overexpression with loss of Ink4a/Arf transforms bone marrow stromal cells into osteosarcoma accompanied by loss of adipogenesis. Oncogene. 2010;29:5687-99.

16. Toguchida J, Ishizaki K, Sasaki MS, Nakamura Y, Ikenaga M, Kato M, et al. Preferential mutation of paternally derived RB gene as the initial event in sporadic osteosarcoma. Nature. 1989;338:156-8.

17. Freeman SS, Allen SW, Ganti R, Wu J, Ma J, Su X, et al. Copy number gains in EGFR and copy number losses in PTEN are common events in osteosarcoma tumors. Cancer. 2008;113:1453-61.
18. Mohseny AB, Tieken C, van der Velden PA, Szuhai K, de Andrea C, Hogendoorn PC, et al. Small deletions but not methylation underlie CDKN2A/p16 loss of expression in conventional osteosarcoma. Genes Chromosomes Cancer. 2010;49:1095-103.

19. Forbes SA, Beare D, Gunasekaran P, Leung K, Bindal N, Boutselakis $\mathrm{H}$, et al. COSMIC: exploring the world's knowledge of somatic mutations in human cancer. Nucleic Acids Res. 2015;43: D805-811.

20. Craven SE, Bredt DS. PDZ proteins organize synaptic signaling pathways. Cell. 1998;93:495-8.

21. Roberts S, Delury C, Marsh E. The PDZ protein discs-large (DLG): the 'Jekyll and Hyde' of the epithelial polarity proteins. FEBS J. 2012;279:3549-58.

22. Ray P, De A, Min JJ, Tsien RY, Gambhir SS. Imaging tri-fusion multimodality reporter gene expression in living subjects. Cancer Res. 2004;64:1323-30.

23. Dunn HA, Ferguson SS. PDZ protein regulation of G proteincoupled receptor trafficking and signaling pathways. Mol Pharmacol. 2015;88:624-39.

24. Hooks SB, Waldo GL, Corbitt J, Bodor ET, Krumins AM, Harden TK. RGS6, RGS7, RGS9, and RGS11 stimulate GTPase activity of Gi family G-proteins with differential selectivity and maximal activity. J Biol Chem. 2003;278:10087-93.

25. van Dam TJ, Bos JL, Snel B. Evolution of the Ras-like small GTPases and their regulators. Small GTPases. 2011;2:4-16.

26. Ross EM, Wilkie TM. GTPase-activating proteins for heterotrimeric $\mathrm{G}$ proteins: regulators of $\mathrm{G}$ protein signaling (RGS) and RGS-like proteins. Annu Rev Biochem. 2000;69:795-827.

27. Stramer B, Wood W, Galko MJ, Redd MJ, Jacinto A, Parkhurst $\mathrm{SM}$, et al. Live imaging of wound inflammation in Drosophila embryos reveals key roles for small GTPases during in vivo cell migration. J Cell Biol. 2005;168:567-73.

28. Coleman ML, Marshall CJ, Olson MF. RAS and RHO GTPases in G1-phase cell-cycle regulation. Nat Rev Mol Cell Biol. 2004;5:355-66.

29. Woods DF, Hough C, Peel D, Callaini G, Bryant PJ. Dlg protein is required for junction structure, cell polarity, and proliferation control in Drosophila epithelia. J Cell Biol. 1996;134:1469-82.

30. Walkley CR, Qudsi R, Sankaran VG, Perry JA, Gostissa M, Roth SI, et al. Conditional mouse osteosarcoma, dependent on p53 loss and potentiated by loss of $\mathrm{Rb}$, mimics the human disease. Genes Dev. 2008;22:1662-76.

31. Chen Y, Di Grappa MA, Molyneux SD, McKee TD, Waterhouse $\mathrm{P}$, Penninger JM, et al. RANKL blockade prevents and treats aggressive osteosarcomas. Sci Transl Med. 2015;7:317ra197. 